\title{
AN EXAMPLE OF SLOW DECAY OF THE SOLUTION OF THE INITIAL-BOUNDARY VALUE PROBLEM FOR THE WAVE EQUATION IN UNBOUNDED REGIONS
}

\author{
BY E. C. ZACHMANOGLOU
}

Communicated by P. D. Lax, March 9, 1964

Let $D$ be a domain in the 3-dimensional Euclidean space $E_{3}$ and let $\$$ be its boundary. Consider the initial-boundary value problem for the wave equation

$$
\begin{aligned}
\nabla^{2} u-u_{t t} & =0, \quad x \in \mathscr{D}, \quad t>0, \\
u(x, 0) & =f(x), \quad u_{t}(x, 0)=g(x), \quad x \in \mathscr{D}, \\
u(x, t) & =0, \quad x \in \mathbb{B}, \quad t>0,
\end{aligned}
$$

where $x=\left(x_{1}, x_{2}, x_{3}\right)$ and $f$ and $g$ are functions defined in D. It is well known that if $D=E_{3}$ and the initial data $f$ and $g$ have compact support then, at each point $x$, the solution $u(x, t)$ is zero after a finite time.

Morawetz [1] showed that if $D$ is the exterior of a smooth bounded star-shaped body and the initial data have compact support then $u$ at each fixed point decays at least as fast as $t^{-1}$. Zachmanoglou [2] showed that the result of Morawetz is true even when the boundary $B$ extends to infinity and the initial data do not have compact support but they satisfy certain conditions at infinity.

Lax and Phillips [3] showed that if $D$ is the region exterior to a finite number of finite bodies then, at each point, $u$ goes to zero. They showed that this is also true when the Dirichlet boundary condition (3) is replaced by the Newmann boundary condition

$$
\frac{\partial u}{\partial n}(x, t)=0, \quad x \in \Theta, t>0 .
$$

Lax, Morawetz and Phillips [4] combined the result of Morawetz with the methods of Lax and Phillips to show that $u$ at each point decays exponentially when $D$ is the region exterior to a bounded smooth star-shaped body and the initial data have compact support. It is the purpose of this note to show that this result is not generally true when the boundary $B$ extends to infinity and has a corner, even though the complement of $D$ is star-shaped.

Let $D$ be a domain bounded by two planes intersecting at an angle 
$\alpha$. When $\alpha=\pi / k, k=1,2, \cdots$, the solution of the initial-boundary value problem (with either of the boundary conditions (3) or (4)) is easily obtained using the reflection principle. It is the sum of the direct wave and $2 k-1$ reflections. If the supports of the initial data $f$ and $g$ are contained in a sphere with center at the origin $x=0$ (located on the line of intersection of the two planes) and radius $R$ then, at each point $x, u(x, t)=0$ when $t>|x|+R$. When $\alpha \neq \pi / k$ the solution is more complicated.

Let $\rho, \phi, z$ be cylindrical coordinates with the $z$-axis coinciding with the line of intersection of the two planes, one plane at $\phi=0$ and the other at $\phi=\alpha$. The following solution of the initial-boundary value problem (1), (2), (3) or (4) with $f=0$ was derived from the solution of the "pulse" problem obtained by Oberhettinger [5]:

$$
\begin{aligned}
& u(x, t)=\frac{1}{4 \pi t} \int_{D} \sum_{n=n_{1}}^{n_{2}} \delta\left(t-\widetilde{R_{n}^{-}}\right) g\left(x^{\prime}\right) d V^{\prime} \\
& \mp \frac{1}{4 \pi t} \int_{\mathscr{D}} \sum_{m=m_{1}}^{m_{2}} \delta\left(t-R_{m}^{+}\right) g\left(x^{\prime}\right) d V^{\prime} \\
& -\frac{1}{8 \pi \alpha} \int_{\mathscr{D}} \frac{F\left(\alpha, \phi-\phi^{\prime}, \beta\right) \mp F\left(\alpha, \phi+\phi^{\prime}, \beta\right)}{\rho \rho^{\prime} \sinh \beta} \\
& \cdot H\left[t^{2}-\left(\rho+\rho^{\prime}\right)^{2}-\left(z-z^{\prime}\right)^{2}\right] g\left(x^{\prime}\right) d V^{\prime}
\end{aligned}
$$

where $\rho, \phi, z\left(\rho^{\prime}, \phi^{\prime}, z^{\prime}\right)$ are the cylindrical coordinates of the point $x\left(x^{\prime}\right) ; \delta(t)$ is the Dirac $\delta$-function; $H(t)$ is the Heaviside unit function ( $=0$ if $t<0$ and $=1$ if $t \geqq 0$ );

$$
\begin{aligned}
& R_{n}^{-}=\left[\rho^{2}+\rho^{\prime 2}+\left(z-z^{\prime}\right)^{2}-2 \rho \rho^{\prime} \cos \left(\phi-\phi^{\prime}+2 \alpha n\right)\right]^{1 / 2} ; \\
& R_{m}^{+}=\left[\rho^{2}+\rho^{\prime 2}+\left(z-z^{\prime}\right)^{2}-2 \rho \rho^{\prime} \cos \left(\phi+\phi^{\prime}+2 \alpha m\right)\right]^{1 / 2} ; \\
& n_{1}=-\left[\frac{\pi+\phi-\phi^{\prime}}{2 \alpha}\right], \\
& n_{2}=\left[\frac{\pi-\phi+\phi^{\prime}}{2 \alpha}\right], \\
& m_{1}=-\left[\frac{\pi+\phi+\phi^{\prime}}{2 \alpha}\right], \\
& m_{2}=\left[\frac{\pi-\phi-\phi^{\prime}}{2 \alpha}\right],
\end{aligned}
$$

where $[t]=$ largest integer less than or equal to $t$; 


$$
\begin{aligned}
2 \rho \rho^{\prime} \cosh \beta= & t^{2}-\rho^{2}-\rho^{\prime 2}-\left(z-z^{\prime}\right)^{2} \\
F(\alpha, \theta, \beta)= & \frac{\sin \left[\frac{\pi}{\alpha}(\pi-\theta)\right]}{\cosh \left(\frac{\pi}{\alpha} \beta\right)-\cos \left[\frac{\pi}{\alpha}(\pi-\theta)\right]} \\
+ & \frac{\sin \left[\frac{\pi}{\alpha}(\pi+\theta)\right]}{\cosh \left(\frac{\pi}{\alpha} \beta\right)-\cos \left[\frac{\pi}{\alpha}(\pi+\theta)\right]}
\end{aligned}
$$

The top sign (-) corresponds to the boundary condition (3) while the bottom sign $(+)$ corresponds to the boundary condition (4).

The terms in the first and second line of equation (5) represent the direct and reflected waves while the third line represents the diffracted wave. We assume from now on that $g$ has compact support. Then it is easily seen that at each point $x$ the direct and reflected waves are zero after a finite time. When $\alpha=\pi / k$, then $F(\pi / k, \theta, \beta)=0$ so that there is no diffracted wave. When $\alpha \neq \pi / k$ the diffracted wave is generally present and we will show that at each point it may decay like a negative power of $t$ depending on the angle $\alpha$.

In particular let $\alpha=2 \pi /(2 k+1)$. Consider first the case of the boundary condition (3). Then for fixed $x$ with $\phi=\alpha / 2=\pi /(2 k+1)$ and sufficiently large $t$,

$$
\begin{aligned}
u(\rho, \alpha / 2, z, t) & =(-1)^{k+1} \frac{2 k+1}{4 \pi^{2}} \int_{D} \frac{\cos \left[(k+1 / 2) \phi^{\prime \prime}\right] \cosh [(k+1 / 2) \beta]}{\cosh ^{2}[(k+1 / 2) \beta]-\sin ^{2}\left[(k+1 / 2) \phi^{\prime \prime}\right]} \\
& \quad \frac{g\left(x^{\prime}\right)}{\rho \rho^{\prime} \sinh \beta} d V^{\prime}
\end{aligned}
$$

where $\phi^{\prime \prime}=\phi^{\prime}-\alpha / 2$. In view of equation (6) it follows easily that in general $u(\rho, \alpha / 2, z, t)$ decays like $t^{-(2 k+3)}$.

For the case of the boundary condition (4), $u(\rho, 0, z, t)$ is given by the right-hand side of equation (7) with $\phi^{\prime \prime}$ replaced by $\phi^{\prime}$. Hence in general $u(\rho, 0, z, t)$ decays again like $t^{-(2 k+3)}$.

\section{BIBLIOGRAPHY}

1. C. S. Morawetz, The limiting amplitude principle, Comm. Pure Appl. Math. 15 (1962), no. 3. 
2. E. C. Zachmanoglou, The decay of solutions of the initial-boundary value problem for the wave equation in unbounded regions, Arch. Rational Mech. Anal. 14 (1963), 312325.

3. P. D. Lax and R. S. Phillips, The wave equation in exterior domains, Bull. Amer. Math. Soc. 68 (1962), 47-49.

4. P. D. Lax, C. S. Morawetz and R. S. Phillips, The exponential decay of solutions of the wave equation in the exterior of a star-shaped obstacle, Bull. Amer. Math. Soc. 68 (1962), 593-595.

5. $\mathrm{F}$. Oberhettinger, On the diffraction and reflection of waves and pulses by wedges and corners, J. Res. Nat. Bur. Standards 61 (1958), no. 5, 343-365.

Purdue University 